\title{
Procalcitonin and C-Reactive Protein as a Specific Marker of Sepsis in Patients Undergoing Cardiac Surgery with Cardiopulmonary Bypass
}

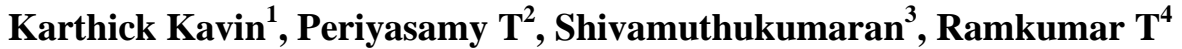 \\ MCh Cardiovascular and Thoracic Surgery Resident, Sri Ramachandra Medical College, Sri Ramachandra University, Porur, Chennai, \\ Tamilnadu-600116, India
}

\begin{abstract}
Introduction: The heart lung machine is perhaps the most important contribution to the cardiac surgery in last century. Artificial surface of cardiopulmonary bypass causes various inflammation processes which will make difficult to differentiate from post operative sepsis. Aim of the study to find out specific marker of sepsis with Procalcitonin (PCT) and C-reactive protein (CRP) in patients undergoing cardiac surgery with cardiopulmonary bypass. Materials and methods, 35 patients of rheumatic heart disease undergoing cardiac surgery with cardiopulmonary bypass who's procalcitonin and C-reactive protein was recorded. Results showed initial increase in level of PCT and CRP, but on second postop day level of PCT significantly dropped to normal in patients without systemic inflammatory response syndrome when compared with patients with SRIS P value $<0.01$. The level of CRP was elevated throughout hospital stay. This shows PCT was a specific marker for sepsis.
\end{abstract}

Keywords: Procalcitonin, C-reactive protein, cardiopulmonary bypass, Cardiac surgery

\section{Introduction}

The heart lung machine is perhaps the most important contribution to the cardiac surgery in last century. The heartlung machine replaces the function of the heart and lung to provide a bloodless, motionless surgical field for the surgeon. This apparatus was designed to perform the function of the both the human heart and the lungs allowing surgeons to suspend normal circulation to repair the heart defects.

Cardio pulmonary bypass, it is impossible to determine who first conceived the idea of diverting the blood outside the body to oxygenator and pumping back to body. Without doubt JOHN GIBBON, his pioneering experimental work at Massachusetts general hospital in late 1930 was a great contribution for the development of cardiopulmonary bypass. During total cardiopulmonary bypass a number of physiologic variables are under direct external control, in contrast to the situation in intact human. There include total systemic blood flow "cardiac output" input pressure waveform, arterial oxygenation, temperature, etc.

One of the main variables is that process of inflammation, incited in large part by organism recognizing the foreign surface across which blood passes on "nonself". Finding out infection in postoperative patients were difficult. As Creactive protein level and leucocytes counts generally increase postoperatively. Clinical deterioration comes once the systemic inflammatory response syndrome advances. Specific markers need to differentiate sepsis and immediate post operative inflammation in patients undergoing cardiac surgery with cardiopulmonary bypass.

Measurement of procalcitonin can be used as a marker of severe sepsis caused by bacteria. Procalcitonin has the greatest sensitivity (85\%) and specificity (91\%) for differentiating patients with systemic inflammatory response syndrome (SIRS) from those with sepsis and post operative inflammation process. The level of procalcitonin in the blood stream of healthy individuals is: $0.15-2 \mu \mathrm{g} / \mathrm{mL}$ (Half life of Procalcitonin: 25-30 hrs). C-reative protein is one of the markers for infection, but not in postoperative patients. Post operative C-reative protein elevation is common. Associated investigation like erythrocytes sedimentation rate and total count were done to find out post operative sepsis.

\section{Aim}

To find out specific marker of sepsis with procalcitonin and C-reactive protien in patients undergoing cardiac surgery with cardiopulmonary bypass

\section{Literature Survey}

Maurice Beghetti Peter et,al[1]. The study measured levels of procalcitonin, interleukin 6 , and C-reactive protein preoperatively, $6 \mathrm{hrs}, 1,3$ and 5 days after cardiopulmonary bypass in 25 children undergoing cardiac surgery. Cardiopulmonary bypass induced a transient increase in procalcitonin, with a peak at $24 \mathrm{~h}$, with a median of 1.13 $\mu \mathrm{g} / \mathrm{ml} \pm 2.25 \mu \mathrm{g} / \mathrm{ml}$, and a $\mathrm{p}$ value of less than 0.001 . The value had returned to normal in the majority of the children by the third day after surgery. Peak values correlated with the duration of cardiopulmonary bypass, with a r-value of 0.58 and a $p$ value of 0.003 , cross-clamp time with a r-value of 0.62 and a $p$ value of 0.001 , days of mechanical ventilation with a r-value of 0.62 and a $p$ value of 0.001 , days of stay in intensive care, with a r-value of 0.68 , and a $p$ value of 0.0003 . The value returned to normal after 3 days in $83 \%$ of the patients. Levels of interleukin 6 and C-reactive protein also increased significantly after surgery and remained elevated for up to 5 days. Thus, in contrast to other markers, levels of procalcitonin in the serum are only slightly and transiently influenced by cardiopulmonary bypass, and may prove to be useful in the early recognition of an infection subsequent to cardiopulmonary bypass. 


\section{International Journal of Science and Research (IJSR) \\ ISSN (Online): 2319-7064}

Index Copernicus Value (2015): 78.96 | Impact Factor (2015): 6.391

A Aouifi et,al[2]. Serum PCT and C-reactive protein concentrations were measured before operation, at the end of surgery and daily until postoperative day 8 . Serum PCT concentrations increased, irrespective of the type of cardiac surgery, with maximum concentrations on day 1.Serum PCT concentrations remained less than $5 \mathrm{ng}$ ml-1 in all patients. Concentrations returned to normal by day 5 in all patients. Samples for PCT and CRP measurements were obtained from 10 other patients with postoperative complications. The increase in serum PCT was significantly greater in SIRS (peak PCT 1.79 (1.64) n/ ml-1 vs. 0.34 (0.32) ng ml-1 in patients without SIRS. Serum PCT concentrations ranged from 6.2 to $230 \mathrm{n} / \mathrm{ml}-1$ in sepsis patients. Serum CRP concentrations increased in all patients, with no differences between groups. The postoperative increase in CRP lasted longer than that of PCT. The study conclude that SIRS induced by cardiac surgery, with and without CPB, influenced serum PCT concentrations with a moderate and transient postoperative peak on the first day after operation. A postoperative serum PCT concentration of more than $5 \mathrm{ng}$ ml-1 is highly suggestive of a postoperative complication.

Michael Meisner et, al[3].The conducted study to find out the specific marker for sepsis in trauma patients. In that study 90 consecutive patients included. Procalcitonin and Creactive protein level were checked serially. Initially level of Procalcitonin and C-reactive protein were high for two days, but procalcitonin showed significant decrease in level compared to C-reactive protein in non sepsis patients. But patients with sepsis showed a significant increase in level even after five days.

Christoph Sponholz, et al[4]. Systemic inflammatory response syndrome is common after surgery, and it can be difficult to discriminate between infection and inflammation. Author performed a review of the literature with the aims of describing the evolution of serum procalcitonin (PCT) levels after uncomplicated cardiac surgery, characterizing the role of PCT as a tool in discriminating infection, identifying the relation between PCT, organ failure, and severity of sepsis syndromes, and assessing the possible role of PCT in detection of postoperative complications and mortality. He performed a search on MEDLINE using the keyword 'procalcitonin' crossed with 'cardiac surgery,' 'heart,' 'postoperative,' and 'transplantation.' Uncomplicated cardiac surgery induces a postoperative increase in serum PCT levels. Peak PCT levels are reached within 24 hours postoperatively and return to normal levels within the first week. This increase seems to be dependent on the surgical procedure and on intraoperative events. Although PCT values reported in infected patients are generally higher than in non-infected patients after cardiac surgery, the cutoff point for discriminating infection ranges from $1-5 \mathrm{ng} / \mathrm{ml}$, and the dynamics of PCT levels over time may be more important than absolute values. PCT is superior to $\mathrm{C}$ reactive protein in discriminating infections in this setting. PCT levels are higher with increased severity of sepsis and the presence of organ dysfunction/failure and in patients with a poor outcome or in those who develop postoperative complications. PCT levels typically remain unchanged after acute rejection but increase markedly after bacterial and fungal infections. Systemic infections are associated with greater PCT elevation than is local infection. Viral infections are difficult to identify based on PCT measurements. The dynamics of PCT levels, rather than absolute values, could be important in identifying patients with infectious complications after cardiac surgery. PCT is useful in differentiating acute graft rejection after heart and/or lung transplantation from bacterial and fungal infections.

Gian Paolo Castelli et.al[5]. In his study one hundred and fifty adult intensive care unit patients were observed consecutively over a period of 10 days. Procalcitonin, Creative protien and infection parameters were compared among the following groups. Procalcitonin and C-reative protein concentrations were higher in patients in whom infection was diagnosed at comparable levels of organ dysfunction. C-reative protein levels were near their maximum already during lower SOFA scores, whereas maximum Procalcitonin concentrations were found at higher score levels (SOFA score > 12). Procalcitonin and C-reative protein concentrations were $1.58 \mathrm{ng} / \mathrm{ml}$ and $150 \mathrm{mg} / \mathrm{l}$ in patients with sepsis, $0.38 \mathrm{ng} / \mathrm{ml}$ and $51 \mathrm{mg} / \mathrm{l}$ in the SIRS patients. The study concluded that kinetics of both parameters were also different, and Procalcitonin concentrations reacted more quickly than $\mathrm{C}$-reative protein.

\section{Materials and Methods}

Thirty five consecutive patients who were undergoing cardiac surgery above age group of 18years and with rheumatic heart disease were included in the study.

Operative Technique: All patients were anesthetized with the standard protocol. After end tracheal intubation, mechanical ventilation was started with oxygen and nitrous oxide. Then the heart was approached through a standard median sternotomy. Heparin $400 \mathrm{IU} / \mathrm{kg}$ was administered intra venous then the ascending aorta SVC and IVC were cannulated. Cardiopulmonary bypass(CPB) was started when the activated clotting time reached more than 400 seconds using non-pulsatile pump flow rate of $2-2.31 / \mathrm{m} 2$ /min. moderate hemodilution with a crystalloid prime moderate systemic hypothermia (to a lowest temperature of $28^{\circ} \mathrm{c}$ ) were used. After aortic cross clamping, myc) were used. After aortic cross clamping, myocardial protection was achieved with intermittent antegrade cold blood cardioplegia through the aortic root till the cardiac arrest occurred and repeated every 22-25 minutes on the return of electrical activity of the heart. Hematocrit concentration (HCT) was maintained between $20 \%-25 \%$ with addition of blood is necessary. Patient was rewarmed to $37^{\circ} \mathrm{c}$. Separation from CPB was accomplished with injection dobutamine $(5 \mathrm{mcq} / \mathrm{kg} / \mathrm{min})$. Heparinization was reversed with injection protamine sulphate $(1-1.3 \mathrm{mg}$ for $100 \mathrm{IU}$ of heparin administered. The baseline arterial sample was collected from patients before and after intubation. First and second day samples were collected from patient in ICU.

\section{Results/Discussion}

Systemic inflammatory response syndrome is common after surgery, and it can be difficult to discriminate between infection and inflammation. After cardiac surgery, the diagnosis of infection remains difficult. Currently available clinical and biological variables, such as CRP and leukocyte

\section{Volume 6 Issue 7, July 2017 www.ijsr.net}




\section{International Journal of Science and Research (IJSR) \\ ISSN (Online): 2319-7064}

Index Copernicus Value (2015): 78.96 | Impact Factor (2015): 6.391

count, are not always conclusive in distinguishing between infection and postoperative SIRS. PCT has been proposed as a specific marker of infection. Because of the postoperative SIRS triggered by cardiac surgery, it remains unknown whether PCT is useful for diagnosis of infection after cardiac surgery. Our study shows that serum concentration

\section{PCT}

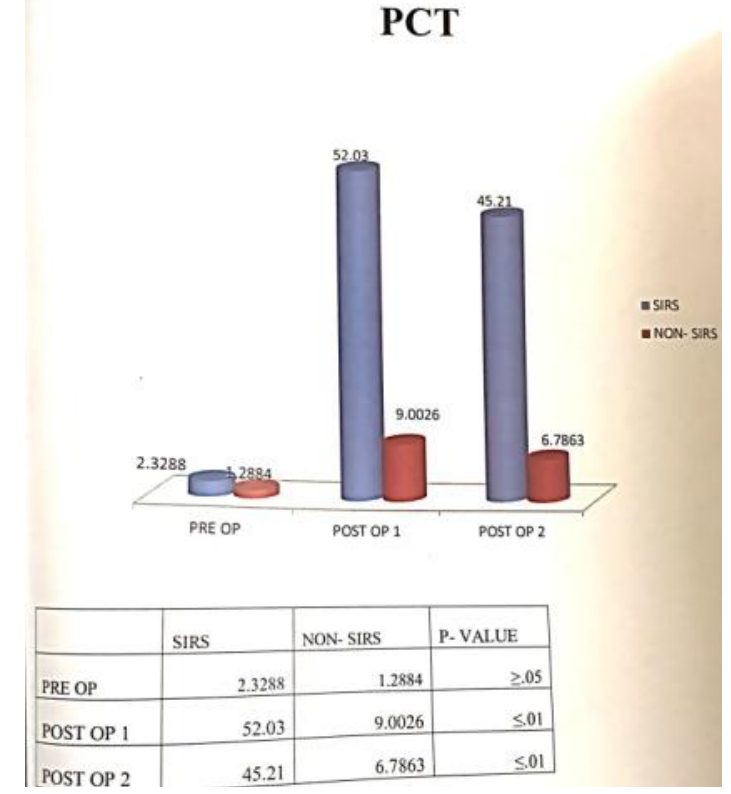

This study includes a total of 35 patients undergoing cardiopulmonary bypass, 16 males and 19 females. The values of preoperative PCT and CRP in patients with/without SIRS were recorded. Statistical analysis was done with IBM.SPSS statics software 23.0 version. To describe about data descriptive statistics frequency analysis, percentage analysis were used to categorical variables and the mean and SD were used for continuous variables. To find the significant difference between the two variables in independent groups T-test was used. Preoperatively PCT elevated to peak of $2.32 \mathrm{ng} / \mathrm{ml}$ with SIRS and $1.28 \mathrm{ng} / \mathrm{ml}$ in without SIRS. Results showed significant decrease in PCT level on second postoperative when compared to first postoperative day $\mathrm{P}$ value of $\leq 0.01$ in both groups.

PCT values peaked on day 1 in 16 patients (>30ng/l). These patients are observed with longer pump time and cross clamp time than 80 mins. This peak is followed by a rapid normalization by day 2 in almost 16 patients. The increase in serum PCT concentration after cardiac surgery appeared to be related to postoperative SIRS, irrespective of the surgical technique. These patients were observed significantly higher CPB time and cross clamp time. This correlation can be explained by bacterial translocation in intestinal tract. The longer the period of non- pulsatile flow during cardio pulmonary bypass, the greater possibility of intestinal barrier disruption and bacterial translocation that can be measured by endotoxemia. Endotoxin is a potent stimulator of PCT production. It also correlates with age, total counts, and duration of ventilation and length of ICU stay.

During CPB endotoxin translocation caused by a transient or more prolonged period of intestinal hypoperfusion is widely documented and this endotoxin release is associated with an increase in TNF $\alpha$, IL- 6 and IL- 8 concentrations. We speculate that postoperative inflammatory cascade is of PCT and CRP increased over normal values after cardiac surgery with the use of CPB. PCT seems to be an important marker of impending complications after cardiac surgery particularly when conventional clinical and biological signs can be difficult to interpret.

\section{CRP}
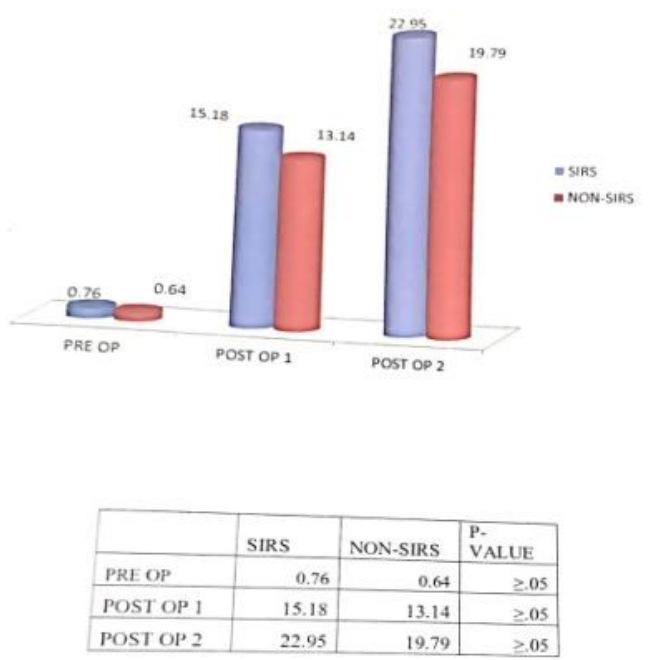

probably responsible for the increase in serum PCT after CPB. Serum CRP concentrations increased markedly in postoperative period, regardless of the type of cardiac surgery. The synthesis of this acute phase protein may not be triggered by $\mathrm{CPB}$ itself, but is probably related to surgical trauma. CRP is a very poor marker of postoperative complications after cardiac surgery; indeed, because of its prolonged increase after operation, serum CRP seems to be less useful than serum PCT for detection of impending postoperative complications.

\section{Conclusion}

PCT is a better marker of sepsis than CRP. The course of PCT shows a closer correlation than that of CRP with the severity of infection and organ dysfunction. It was clear that patients with elevated PCT on $2^{\text {nd }}$ post operative day had increased ICU stay and was put on aggressive sepsis management. Thus PCT helps to reduce mortality and morbidity in cardiac surgery especially in rheumatic patients due to sepsis.

\section{References}

[1] Beghetti $\quad \mathrm{M}^{1}$, Rimensberger $\quad \mathrm{PC}$, Kalangos A, Habre W, Gervaix A.Kinetics of procalcitonin, interleukin 6 and C-reactive protein after cardiopulmonary-bypass in children. Cardiol Young. 2003 Apr;13(2):161-7.

[2] Aouifi $\mathrm{A}^{1}$, Piriou V, Blanc P, Bouvier H, Bastien O, Chiari P, Rousson R, Evans R, Lehot JJ. Effect of cardiopulmonary bypass on serum procalcitonin and Creactive protein concentrations. $\mathrm{Br} \mathrm{J}$ Anaesth. 1999 Oct;83(4):602-7.

[3] Meisner $\mathbf{M}^{1}$, Adina $\mathrm{H}$, Schmidt J. Correlation of procalcitonin and C-reactive protein to inflammation, complications, and outcome during the intensive care 


\section{International Journal of Science and Research (IJSR) \\ ISSN (Online): 2319-7064}

Index Copernicus Value (2015): 78.96 | Impact Factor (2015): 6.391

unit course of multiple-trauma patients. Crit Care. 2006 Feb;10(1):R1.

[4] Sponholz $\quad C^{1}$, Sakr Y, Reinhart K, Brunkhorst $\quad F$. Diagnostic value and prognostic implications of serum procalcitonin after cardiac surgery: a systematic review of the literature. Crit Care. 2006;10(5):R145.

[5] Gian Paolo Castelli, ${ }^{1}$ Claudio Pognani, ${ }^{1}$ Michael Meisner, ${ }^{2}$ Antonio Stuani, ${ }^{1}$ Daniela Bellomi, ${ }^{3}$ and Laura Sgarbi $^{1}$ Procalcitonin and C-reactive protein during systemic inflammatory response syndrome, sepsis and organ dysfunction. Published online 2004 Jun 10. 